\title{
Impact of primary aberrations on coherent lidar performance
}

\author{
Hu, Qi; Rodrigo, Peter John; Iversen, Theis Faber Quist; Pedersen, Christian
}

\section{Published in:}

Proceedings of SPIE

Link to article, DOI:

$10.1117 / 12.2037179$

Publication date:

2014

Document Version

Publisher's PDF, also known as Version of record

Link back to DTU Orbit

\section{Citation (APA):}

Hu, Q., Rodrigo, P. J., Iversen, T. F. Q., \& Pedersen, C. (2014). Impact of primary aberrations on coherent lidar performance. In Proceedings of SPIE (Vol. 8992). [89920T] SPIE - International Society for Optical Engineering. Proceedings of SPIE - The International Society for Optical Engineering https://doi.org/10.1117/12.2037179

\section{General rights}

Copyright and moral rights for the publications made accessible in the public portal are retained by the authors and/or other copyright owners and it is a condition of accessing publications that users recognise and abide by the legal requirements associated with these rights.

- Users may download and print one copy of any publication from the public portal for the purpose of private study or research.

- You may not further distribute the material or use it for any profit-making activity or commercial gain

- You may freely distribute the URL identifying the publication in the public portal 


\section{Impact of primary aberrations on coherent lidar performance}

Hu, Qi, Rodrigo, Peter John, Iversen, Theis F., Pedersen, Christian

Qi Hu, Peter John Rodrigo, Theis F. Q. Iversen, Christian Pedersen, "Impact of primary aberrations on coherent lidar performance," Proc. SPIE 8992, Photonic Instrumentation Engineering, 89920T (8 March 2014); doi: 10.1117/12.2037179

SPIE. Event: SPIE OPTO, 2014, San Francisco, California, United States 


\title{
Impact of primary aberrations on coherent lidar performance
}

\author{
Qi Hu*a, Peter John Rodrigo ${ }^{\mathrm{a}}$, Theis F.Q. Iversen ${ }^{\mathrm{b}}$ and Christian Pedersen ${ }^{\mathrm{a}}$ \\ ${ }^{a}$ DTU Fotonik, Frederiksborgvej 399, 4000 Roskilde, Denmark; \\ ${ }^{b}$ Windar Photonics A/S, Helgeshøj Alle 16-18, 2630 Taastrup, Denmark
}

\begin{abstract}
In this work we investigate the performance of a monostatic coherent lidar system in which the transmit beam is under the influence of primary phase aberrations: spherical aberration (SA) and astigmatism. The experimental investigation is realized by probing the spatial weighting function of the lidar system using different optical transceiver configurations. A rotating belt is used as a hard target. Our study shows that the lidar weighting function suffers from both spatial broadening and shift in peak position in the presence of aberration. It is to our knowledge the first experimental demonstration of these tendencies. Furthermore, our numerical and experimental results show good agreement.

We also demonstrate how the truncation of the transmit beam affects the system performance. It is both experimentally and numerically proven that aberration effects have profound impact on the antenna efficiency, the optimum truncation of the transmit beam and the spatial sensitivity of a CW coherent lidar system. Under strong degree of aberration, the spatial confinement is significantly degraded. However for SA, the degradation of the spatial confinement can be reduced by tuning the truncation of the transmit beam, which results from the novel finding in this work, namely, that the optimum truncation ratio depends on the degree of SA.
\end{abstract}

Keywords: Remote Sensing, Lidar, Laser Doppler velocimetry

\section{INTRODUCTION}

To harvest the energy efficiently in wind industry, the wind turbines' rotor yaw and blade pitch need to be controlled in response to the wind conditions. However, the accuracy of the conventional wind-sensing instruments like anemometer and wind vanes mounted on top of the turbines is strongly compromised due to wind turbulence, created by the rotor blades. An average yaw errors up to 10 to 15 degrees $^{1}$ can occur. For this reason, laser remote sensing (lidar) technology, which measures wind in front of wind turbines, is an attractive alternative. The yaw error correction can also prolong the turbine lifetime up to $10 \%$ due to reduced mechanical load. ${ }^{2}$

Spatial confinement of the probing beam is a very important parameter for coherent wind lidar designs. To our knowledge, there are no experimental treatment of this topic in the literature, and the existing theories are all focused on diffraction limited system designs. A theoretical analysis by [Rye, 1982 $]^{3}$ did include the aberrations, but only for a fixed beam truncation through the optical transceiver. Thus, this work is dedicated to experimental investigation of how aberration effects induced in the transceiver telescope can affect this beam confinement. Only the influence of SA and astigmatism will be covered here, since coma effects can easily be avoided during experimental alignment procedures. Furthermore, we will illustrate, both numerically and experimentally, how the aberrations affect the antenna efficiency, and the optimum truncation of the transmitted beam.

\section{SETUP AND METHODOLOGY}

In this work, the experimental investigations are based on probing the weighting function of the lidar system using a rotating belt as a hard target. A sketch of the lidar design can be found in Fig. 1. The system is designed such that lens $L_{2}$ is the only component which introduces aberrations. In cw coherent wind lidar systems, a large collection lens $\left(L_{2}\right)$ is necessary to obtain a reasonable signal and spatial confinement of the probing volume. Due to the large size of the collection lens, it often becomes the most expensive optical component in a lidar design, if diffraction limited performance is desired.

*huqi@fotonik.dtu.dk

Photonic Instrumentation Engineering, edited by Yakov G. Soskind, Craig Olson,

Proc. of SPIE Vol. 8992, 89920T · (c) 2014 SPIE · CCC code: 0277-786X/14/\$18 doi: $10.1117 / 12.2037179$

Proc. of SPIE Vol. $899289920 T-1$ 


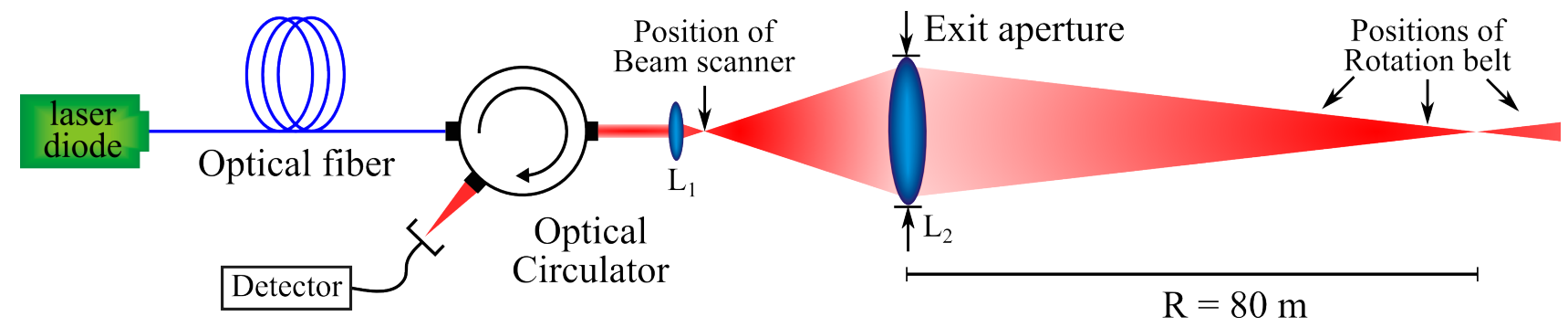

Figure 1: Schematic layout of the lidar system. The light source is a $1550 \mathrm{~nm}$ cw laser and the size of the exit aperture is determined by the diameter of $L_{2}$. A small portion of the laser output $(0.5 \mathrm{~mW})$ and the lidar signal are directed onto the detector inside the circulator.

In the experiments, two $L_{2}$ lenses with different $\mathrm{SA}$ are used, and different $L_{1}$ lenses are used to probe different beam truncations through the transceiver telescope. An overview of different truncation ratios, $\rho$, are listed in table 1. For every $L_{1}$ and $L_{2}$ combinations, the lens separation is adjusted such that the intended probing range, $R=80 \mathrm{~m}$. (As we will see later, the real focus distance might differ from $R$ due to aberrations.) This is achieved by maximizing the lidar signal while tuning the distances between $L_{1}$ and $L_{2}$, the position of the rotating belt is $80 \mathrm{~m}$ after $L_{2}$.

Table 1: Relation between focal lengths of $L_{1}$ and $\rho$

\begin{tabular}{|c|c|c|c|c|c|}
\hline Focal length of $L_{1}, f_{1}$ & $4.6 \mathrm{~mm}$ & $8.1 \mathrm{~mm}$ & $11.3 \mathrm{~mm}$ & $15.6 \mathrm{~mm}$ & $18.8 \mathrm{~mm}$ \\
\hline$\rho$, singlet $L_{2}$ & 1.28 & 0.73 & 0.52 & 0.38 & 0.31 \\
\hline$\rho$, doublet $L_{2}$ & 1.38 & 0.79 & 0.56 & 0.41 & 0.34 \\
\hline
\end{tabular}

A small portion of the diode laser output is tapped within the optical ciculator and is used as the local oscillator (LO) for the heterodyne detection. In the optical circulator, both the backscattered signal from the target and the LO is focused onto the detector. This configuration is commonly referred to as the Wang design. ${ }^{4}$

As shown in table 1 , one of $L_{2}$ is a singlet lens while the other one is a doublet lens with SA corrections. For the singlet $L_{2}$ case the Zernike coefficient for the primary spherical aberration is $Z_{4}^{0}=2.16$, while the doublet $L_{2}$ has almost zero SA, $Z_{4}^{0}=0.0819$. The calculated optical patch difference (OPD) in wavenumbers of the singlet $L_{2}$ is shown in Fig. 2. It gives us a more quantitative description of the aberrations in the lens. The OPD graph is used in all the numerical simulations of the singlet $L_{2}$ case. While in the doublet $L_{2}$ case, the simulations are based on an approximation of zero aberrations.

Our numerical simulations rely on a theoretical prediction of the field or irradiance distribution on the target side of $L_{2}$. The aberration effect can be mathematically incorporated in the theoretical simulation by introducing a phase term, $\phi_{\mathrm{SA}}$ to the truncated Gaussian field at the position of $L_{2}$ :

$$
E(r)=\left\{\begin{array}{cl}
E_{0} \exp \left\{-\frac{r^{2}}{w^{2}}+i \phi(r)+i \phi_{S A}(r)\right\} & \text { for } 0 \leq r \leq r_{L 2} \\
0 & \text { for } r>r_{L 2}
\end{array}, \quad \phi_{S A}=2 \pi * \mathrm{OPD}(r)\right.
$$

where $E_{0}$ is the normalization amplitude, $r$ is the radial coordinate, $\phi(r)$ is the phase of the beam due to field curvature, $\phi_{S A}$ is the phase term induced by the SA. One can "virtually" back propagate the local oscillator from the detector plane to the target plane to simplify the numerical calculations. This is called the target plane formalism, introduced first by Siegmann $(1966)^{5}$ and is known as Siegman's antenna theorem. Quantity $w$ in Eq.(1) is the beam width of the back propagated local oscillator (BPLO) at the position of $L_{2}$. Due to the circular symmetry, the output field on the target plane can be calculated numerically by a simple Fourier-Bessel transformation of Eq.(1) with an appropriate field curvature. ${ }^{3}$ This numerical calculation is proven to be very accurate as demonstrated in $[\mathrm{Q} . \mathrm{Hu} \text { et al. }]^{6}$ 


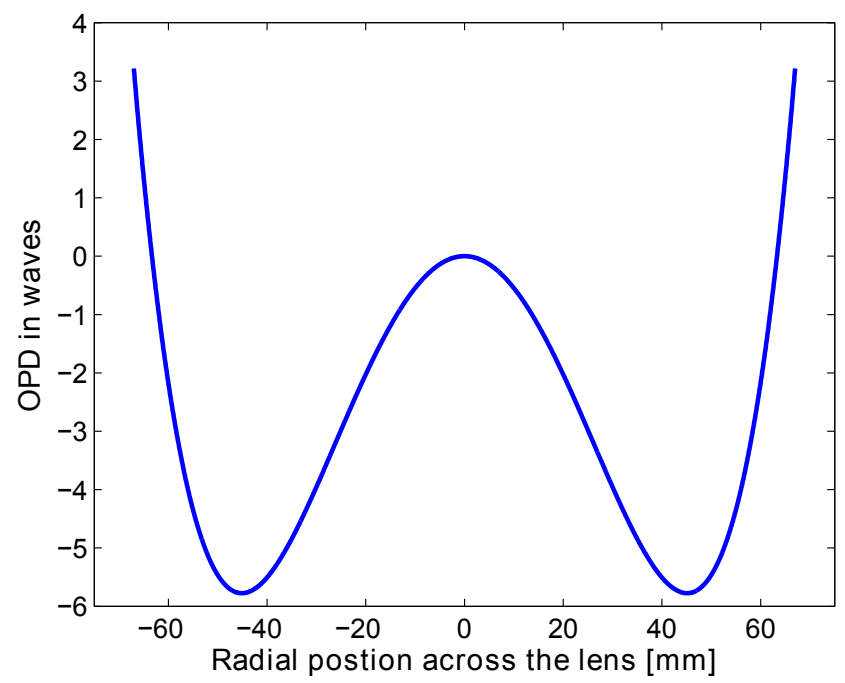

Figure 2: The OPD of the singlet $L_{2}$ used in our experiments. The OPD is generated in Zemax with zero incident angle and circular symmetry.

Applying the target plan formalism antenna efficiency of the lidar system can be calculated as follow: ${ }^{3,5,7}$

$$
\eta_{a}(z)=\frac{\lambda^{2} R^{2}}{A_{r}} \iint I_{\text {target }}^{2}(x, y, z) d x d y
$$

where $\lambda$ is the wavelength, $R$ is the intended imaging or probing range, $A_{r}$ is the area of $L_{2}$ and $I_{\text {target }}$ is the irradiance distribution of the output beam at the target plane, normalized by the total beam power before truncation.

\section{RESULTS AND DISCUSSIONS}

In this section, we will present the obtained results during our work. The measured lidar signals will be compared with numerically simulated antenna efficiency. This comparison should be valid even though we are not simulating the lidar signal itself, because we are only interested in the shape of the weighting functions in different lens configurations. In the numerical model, we do simulate the lens distance separation tuning process mentioned in the methodology. In this way, we ensure the consistency between the experiment and the simulations.

\subsection{Numerical results}

The numerical simulations of the weighting function are shown in Fig. 3, and the figure contains every lens configurations from the experiments. By comparing Fig. 3 a) and b), it is apparent that the overall performance degrades in the presence of SA. Both the peak intensity and the spatial confinement are compromised due to the SA effects. On top of that, the position of the maximum lidar signal response is shifted towards shorter distances than the intended probing range $(80 \mathrm{~m})$. This observation is consistent with the results presented by Rye ${ }^{3}$ although his results only apply to a beam truncation ratio of $\rho=0.8$.

In aberration free systems, a truncation ratio of $\rho \approx 0.8$ is required for optimal lidar performance, ${ }^{7}$ which is also the case in our simulation (green curve in Fig.3b) has a truncation ratio of $\rho=0.79$ ). However, under the influence of SA effects, this is no longer true, see Fig. 3a). The result suggests that the optimal truncation ratio shifts towards a lower value in the presence of SA effects. This behaviour is actually quite reasonable. By reducing the truncation ratio, we are effectively reducing the spherical aberration, experienced by the transmitted beam. Ultimately, the system will approach the diffraction limited case, when the output beam width approaches zero. However, with decreasing beam size the spatial confinement of the lidar will diminish as well. So there must be an optimal truncation ratio for none-zero aberration cases as well. 

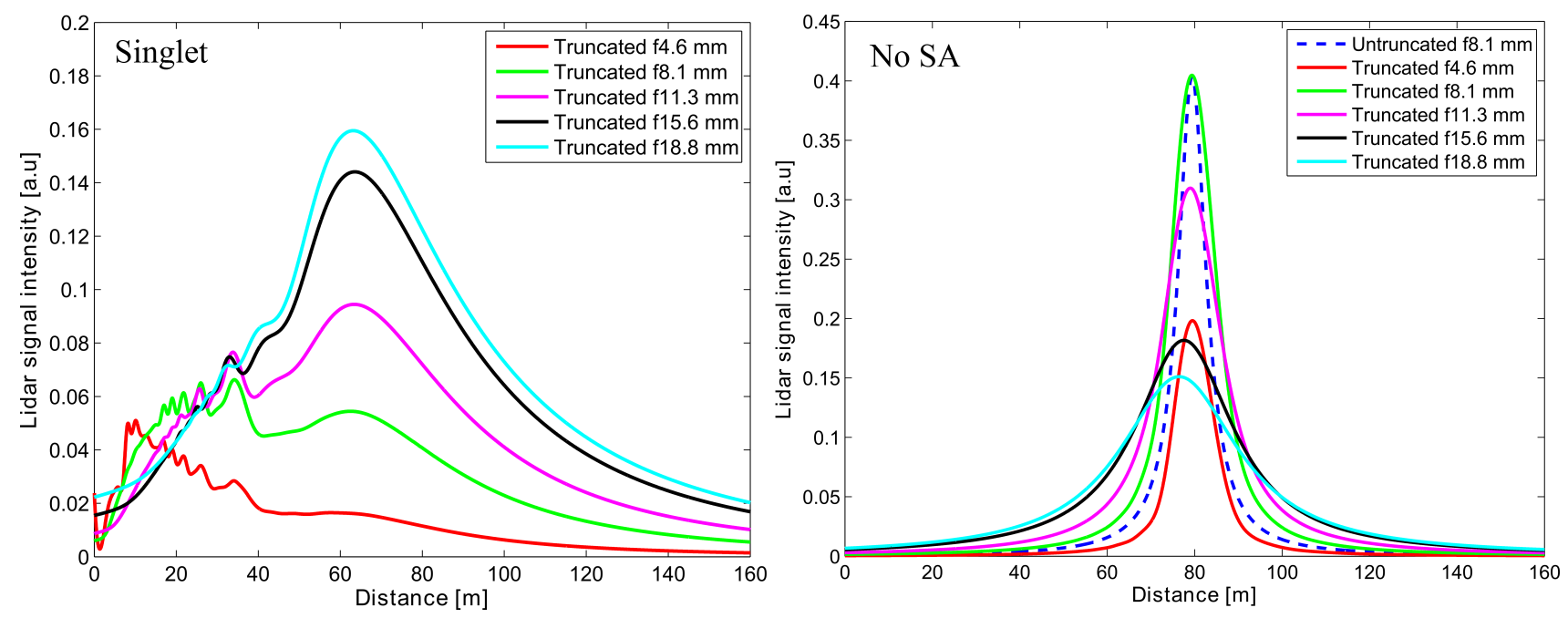

Figure 3: Simulation of the weighting functions for all the transceiver configurations: a) the singlet $L_{2}$ case, b) No SA case. The simulations are acquired using the numerical integration of the fields including the truncation diffraction effects. The blue dashed line in the right figure is the simulated weighting function without truncation effect for $f_{1}=8.1 \mathrm{~mm}$.

Another point worth mention is the inclusion of beam truncation in our simulations. Most models (which assume a Lorentzian profile for the weighting function) do not include this effect to the weighting function shape of a cw coherent lidar. The blue dashed line in Fig. 3 is produced with the same parameters as the green curve, but in the absence of beam truncation. The weighting function width is increased by $57 \%$, when comparing the green curve with the blue dashed line. This indicates that it is essential to include the truncation effect in theoretical calculations of such lidar systems.

\subsection{Experimental results}

As mentioned in the methodology section, that the weighting function of the lidar system is experimentally accessed by measuring the lidar signal of a rotating belt in different positions on the target side of $L_{2}$. In this way, we can probe the spatial response of our system.

The experimental results are presented in Fig. 4 along with their theoretical fits. For a monostatic CW lidar, the weighting function is commonly described by a Lorentzian function under the assumption of an ideal and untruncated Gaussian beam output. ${ }^{8}$ The assumption is adequate for many of the cases shown in Fig. 4. The scattered points in the plots are the measured data points, while the solid lines are Lorentzian fit to these data, when applicable. To our knowledge, no one else has ever conducted an experimental confirmation/investigation of the coherent lidar performance under the influences of aberration effects yet.

The graphs in Fig.4 and Fig. 3 show a good agreement between our simulations and the data. The purple peak at distance $Z=30 \mathrm{~m}$ in Fig. 4a), suggests that even the frequent oscillations in Fig. 3a) are not artificial (one might expect these peaks are introduced due to numerical errors). However, there are minor deviations between the experimental data and the simulations.

One explanation is, that the doublet $L_{2}$ is not completely aberration free. However, this tiny amount of aberration leads to some interesting result in our investigation. If one observes the optimal case (green curve) in Fig.4b) carefully, it actually shows a slight peak shift of the maximum signal response. The intended probing range is at $80 \mathrm{~m}$, while the real probing range is only $78.6 \mathrm{~m}$. The simulated probing range in Fig. 3 is actually $79.4 \mathrm{~m}$. This indicates that even the slightest SA can cause a probing range shift of the lidar system. The fact, that every other plots in Fig. 4 and 3 demonstrate the same and enhanced trends, supports our claim. 

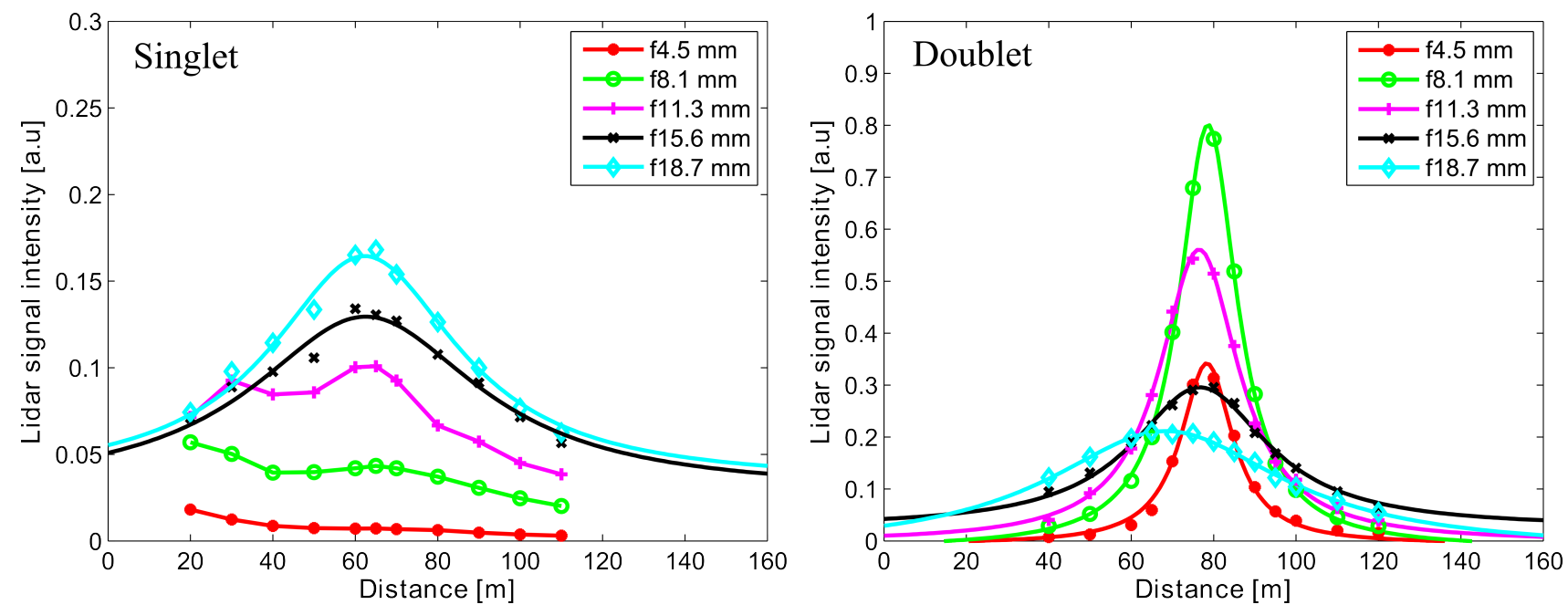

Figure 4: The measured weighting functions for all the transceiver configurations both for the singlet $L_{2}$ case and the doublet $L_{2}$ case. The cyan line represents the optimal $\rho=0.3$ for the singlet case, while the green line corresponds to the optimal $\rho$ of 0.8 for the doublet case. Lorentzian fits of the experimental data (scattered points) were performed when possible.

\subsection{Astigmatism}

Since the weighting function is used as a performance benchmark for the lidar system, a more quantitative comparison between the simulation and data is presented. The optimal lens configurations for the doublet $L_{2}$ case is used as the example. The full width half maximum (FWHM) of the weighting is a direct indication of the spatial resolution of a lidar system. For most applications, high spatial resolutions are desirable (a small FWHM).

The FWHM of the green plot in Fig. 3 is $12.25 \mathrm{~m}$, while the width is measured to be $17.1 \mathrm{~m}$ in our experiment. This deviation of $4.85 \mathrm{~m}$ is partly due to the fact that the doublet $L_{2}$ is not completely SA free, but the main contribution to the deviation is caused by astigmatism in the beam profile, and the astigmatism is not included in the simulations. Normally, if there are equal amount of astigmatism and SA present, the impact of SA effect will dominate. ${ }^{3}$ However, in the doublet $L_{2}$ case, the degree of astigmatism is much larger than SA. Thus, the impact of the astigmatism is visible in our data.

To confirm it, we have measured the beam profile from the optical circulator output. The beam scanner is placed around the beam waist after $L_{1}$ as shown in Fig. 1 , and $f_{1}=18.8 \mathrm{~mm}$ was used for all the beam scanning measurement to reduced measurement uncertainty. (Longer focal length gives a larger beam waist after transmission through the lens.)

An initial result from the beam scanner shows a beam ellipticity of 0.96 , see Fig. 5 . To obtain more reliable data, we have used a complete $\mathrm{M}^{2}$ beam quality analysis system (M2SET-BP209IR, Thorlabs) to map the full $3 \mathrm{D}$ profile of the output beam around the beam waist after $L_{1}$. The same measurement was repeated 10 times to reduce the uncertainties, and the key mean values are as follow; 1) Beam waist in X direction: $21.9 \mu \mathrm{m}, 2$ ) Beam waist in Y direction: $18.2 \mu \mathrm{m}, 3$ ) Beam waist position difference between $\mathrm{X}$ and $\mathrm{Y}$ directions: $0.11 \mathrm{~mm}$.

The beam waist displacement in $\mathrm{X}$ and $\mathrm{Y}$ direction is actually quite large, considering the size of the beam waist. Let us make estimate of this beam waist displacement at the position of $80 \mathrm{~m}$ after $L_{2}$. The parameters of the green curves in the figures: $f_{1}=8.1 \mathrm{~mm}$ and $f_{2}=216 \mathrm{~mm}$. The estimate is based on the thin lens formula, Eq. (3), and the assumption that the beam waist size after $L_{1}$ scales linearly (it is true to first order) with $f_{1}$.

$$
\frac{1}{f}=\frac{1}{s_{1}}+\frac{1}{s_{2}}
$$

where $f$ is the focal length of the lens, $s_{1}$ is object distance to the lens and $s_{2}$ is image distance after the lens. Since the beam focus is $80 \mathrm{~m}$ after $L_{2}$, we get $s_{1}=216.5848 \mathrm{~mm}$ and $s_{2}=80 \mathrm{~m}$, according to Eq.(3). In the 


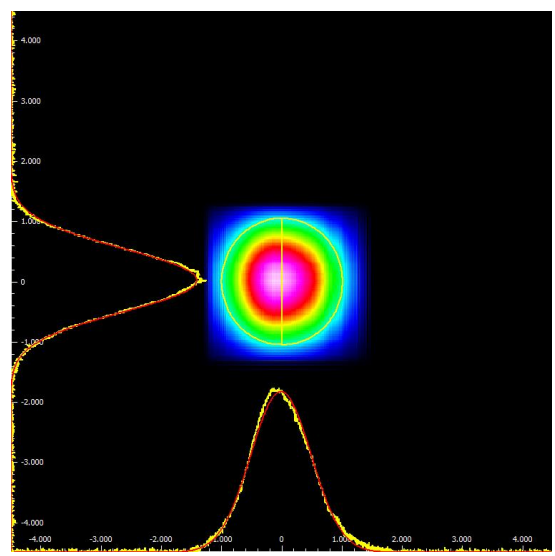

Figure 5: 2D profile of the scanned beam output using $L_{1}$ lens with $f_{1}=18.8 \mathrm{~mm}$, the scanned profile has an ellipticity of 0.96 .

case of $f_{1}=8.1 \mathrm{~mm}$, the beam waist position difference in the two orientations after $L_{1}$ are around $47 \mu \mathrm{m}$, since the displacement scales linearly with $f_{1}$. Now adding the displacement distance to $s_{1}$, we get $s_{2}=87 \mathrm{~m}$ when applying Eq.(3) again. So the beam waist displacement in $\mathrm{X}$ and $\mathrm{Y}$ directions after the doublet $L_{2}$ is around $7 \mathrm{~m}$. This number coincides well with the FWHM deviation between the simulation and the data in Fig. 3 and 4, confirming our claim about astigmatism being the main reason behind the discrepancy in our results.

\section{CONCLUSION}

In this work we have demonstrate experimentally that both SA and astigmatism can have profound impact on the lidar performance in terms of spatial confinement of the probing volume. And we have shown that the results are well in line with the theoretical expectation, both the literature and our own expanded model. A simulation that includes the astigmatism should be implemented in future work in order to improve and confirm our model.

\section{REFERENCES}

1. Pedersen, T. F., Sørensen, N. S., Vita, L., and Enevoldsen, P., "Optimization of Wind Turbine Operation by Use of Spinner Anemometer," Risø Report, Risø-R-1654(EN), August (2008).

2. http://www.nrel.gov/wind/pdfs/39154.pdf

3. Rye, B. J., "Primary aberration contribution to incoherent backscatter heterodyne lidar resturns," Appl. Opt. 21, 839-844 (1982).

4. Wang, J. Y., "Optimal truncation of a lidar transmitted beam," Appl. Opt. 27, 4470-4474 (1988)

5. Siegman, A. E., "The antenna properties of optical heterodyne receivers," Appl. Opt. 5, 1588-1594 (1966).

6. Hu,Q., Rodrigo, P. J., Iversen, T.F.Q., and Pedersen, C., "Investigation of spherical aberration effects on coherent lidar performance," Opt. Express 21, 25670-25676 (2013).

7. Fujii, T., and Fukuchi, T., eds. [Laser Remote Sensing] (CRC Press, 2005).

8. Mann,J., Peña, A., Bingöl, F., Wagner, R., and Courtney, M. S., "Lidar scanning of momentum flux in and above the atmospheric surface layer," J. Atmos. Oceanic Technol. 27, 959-976 (2010). 